\title{
Swift-BAT: the hard-X-ray sky after 66 months of survey
}

\section{La Parola*}

INAF, Istituto di Astrofisica Spaziale e Fisica Cosmica, sez. Palermo

E-mail: laparola@ifc.inaf.it

\section{G. Cusumano, A. Segreto, A. Maselli, P. Romano}

INAF, Istituto di Astrofisica Spaziale e Fisica Cosmica, sez. Palermo

\begin{abstract}
The Burst Alert Telescope (BAT: 14-150 keV) on board of Swift is mainly devoted to the monitoring of a large fraction of the sky (50\%-80\% per day) for the occurrence of Gamma Ray Bursts. This provides the opportunity for a substantial gain of our knowledge of the Galactic and extragalactic sky in the hard X-ray domain. Here we present the third Palermo Swift-BAT hard $\mathrm{X}$-ray catalogue obtained from the analysis of the data relative to the first 66 months of the Swift mission and including 1500 high-energy sources. With a program of soft X-ray follow-up observations and by using archival data we were able to associate a counterpart to most of these high energy emitters: $59 \%$ are extragalactic objects, $20 \%$ are Galactic objects and $9 \%$ are known soft $\mathrm{X}$-ray emitters whose nature has not been determined yet. We compare our catalogue with those obtained from the INTEGRAL-ISGRI data and with the gamma-ray sky as seen by Fermi.
\end{abstract}

8th INTEGRAL Workshop "The Restless Gamma-ray Universe"

September 27-30 2010

Dublin Castle, Dublin, Ireland

\footnotetext{
${ }^{*}$ Speaker.
} 

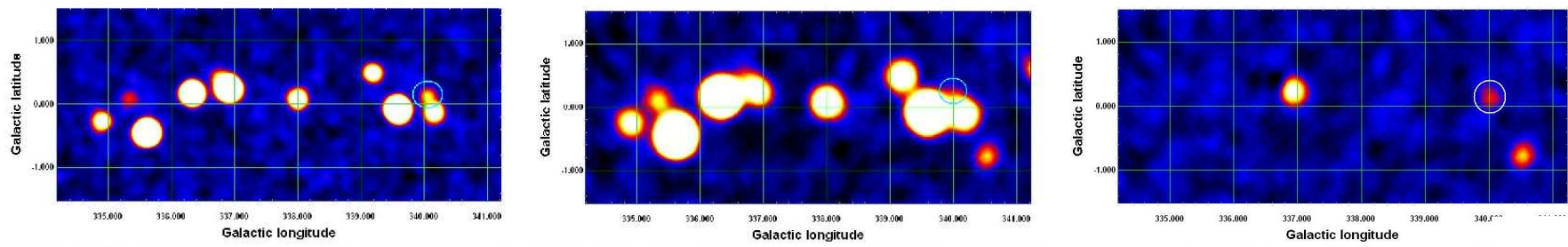

Figure 1: Left: INTEGRAL-IBIS significance map of a crowded field; Center: BAT significance map of the same field; Right: BAT residual significance map. In the three images, the circle marks the position of IGR J16465-4507. The IBIS and the BAT maps have been built integrating over the same survey exposure time.

\section{Introduction}

The Burst Alert Telescope (BAT; [1]) onboard the Swift observatory [2] is a coded-aperture imaging camera operating in the $15-150 \mathrm{keV}$ energy range, whose main goal is the monitoring of a large fraction of the sky for the occurrence of gamma ray bursts (GRBs). While waiting for new GRBs, BAT continuously collects spectral and imaging information from the sky, covering a fraction of between $50 \%$ and $80 \%$ of the sky every day. Together with its large field of view (1.4 steradian half coded) and with its 17 arcmin (full width half maximum) point spread function, this pointing strategy makes this telescope an ideal instrument for hard X-ray surveys (see e.g. [3], [4], [5], [6]).

In order to exploit the survey data, we have developed a code that performs screening, mosaicking, and source detection on data from coded mask instruments [7]. We have thus produced the first two Palermo BAT Catalogues (PBC), from the analysis of the first 39 months [8] and 54 months [9] of the Swift mission. In this paper we present the preliminary results of the analysis of 66 months of BAT survey data.

\section{Building the catalogue}

\subsection{Source detection}

We have analysed the survey data collected between November 2004 and May 2010. We have built significance all-sky maps in three energy bands (15-30 keV, 15-70 keV, and 15-150 keV), and in order to optimize the detection of sources, we have proceeded in three steps.

All-sky significance maps. We have performed a blind search for significance peaks on each of the three maps, with a S/N threshold of 4.8 standard deviations. We obtain $\sim 1400$ source candidates, with $\sim 30$ expected spurious detections.

Residuals significance maps. The crowding of bright sources (as, for exemple in the Galactic center) may hide the presence of close fainter sources. In order to make these fainter source emerge, 

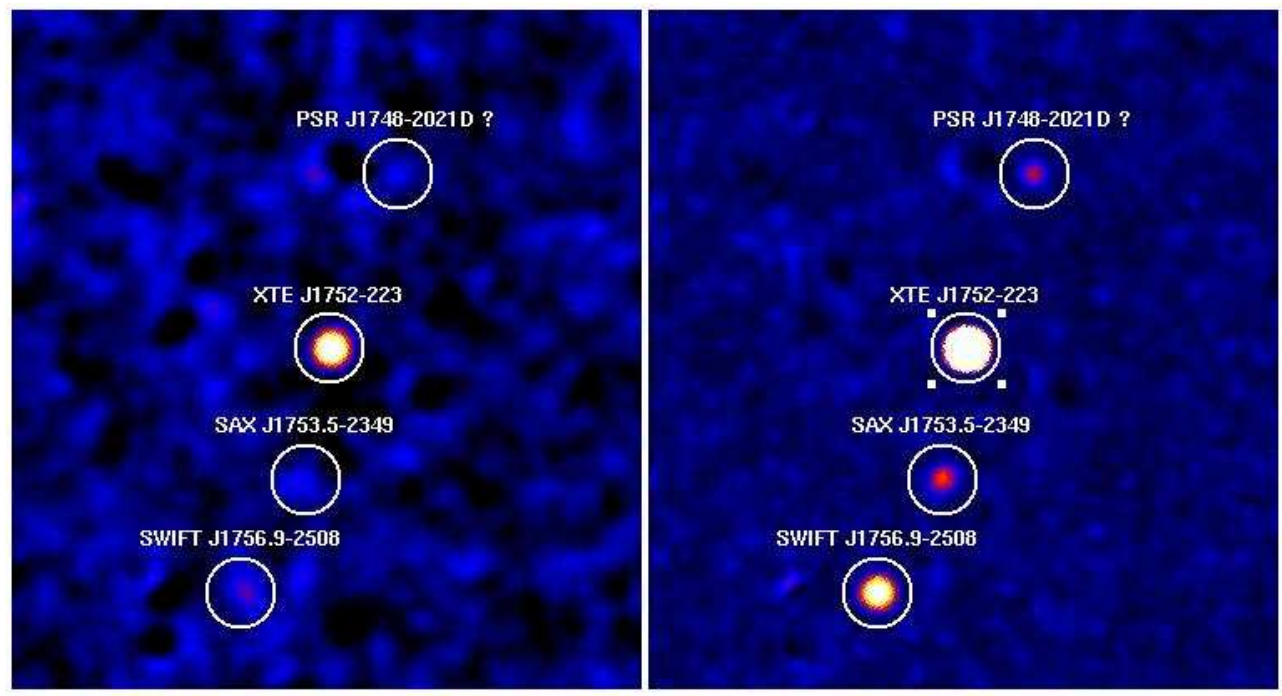

Figure 2: Left: $15-150 \mathrm{keV}$ significance map. Right: best significance map obtained associating to each pixel the best signal-to-noise achievable summing over consecutive time bins.

we have subtracted from the all-sky significance maps all the sources detected in the previous step, and run the detection algorithm on this residual map. Figure 1 illustrates one of these cases: IGR J16465-4507 (clearly detected in the IBIS map, left panel) in the BAT map (central panel) is embedded between IGR J16479-4514 and GX340+0, due to the worse spatial resolution of BAT with respect to IBIS. However, it emerges clearly in the map where these two sources have been subtracted (right panel).

Transient search. Some transient sources may be undetectable in the integrated all-sky map, where their average significance falls below the detection threshold. In order to make these sources emerge, we have built light curves for each sky pixel, at the angular resolution of 2.5 arcmin, with a time resolution of 15 days. We have thus built a "best significance" all-sky map, where we have assigned to each pixel the maximum significance value that can be obtained cumulating the signal of consecutive time bins. A detection algorithm is then run on this map. Figure 2 shows how a region on the Galactic plane appears in the all-sky significance map and in the "best significance" map, with three sources emerging in the latter, where no signal was detected in the former map. Figure 3 shows the light curve (with a bin time of 15 days) of one of these sources, with the significance averaged over the 66 months (magenta line) and the significance achieved integrating along the "best significance" time interval (blue line): the latter is the value reported in the"best significance" map.

The final catalogue of BAT sources contains $\sim 1600$ detections.

\subsection{Source association}

In order to associate a counterpart to the BAT detections, we have followed two methods (for a full description see [9]). 


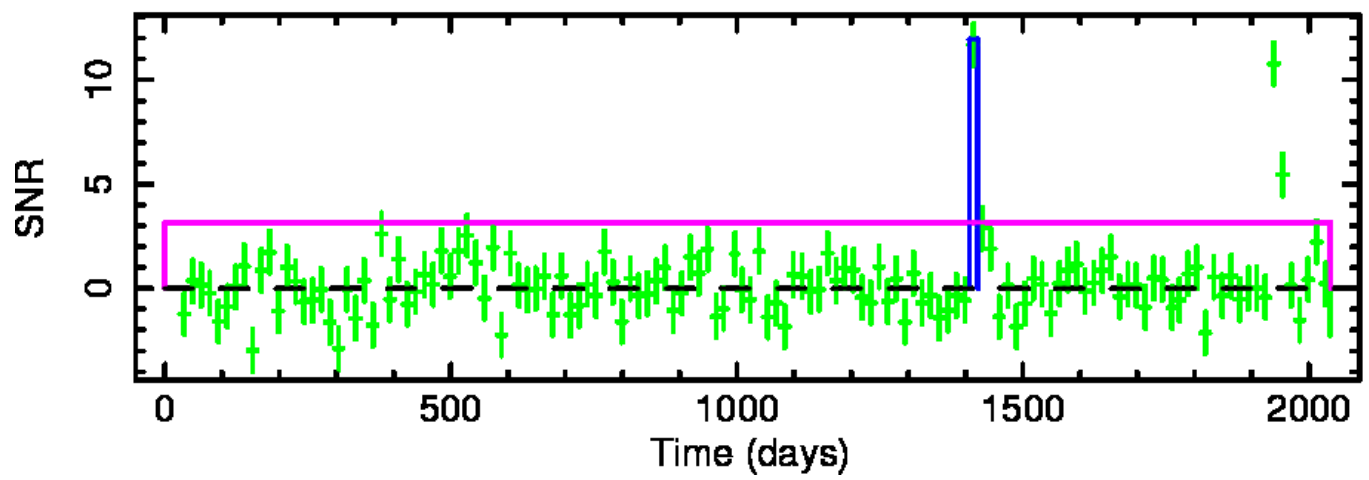

Figure 3: Significance light curve of SAX J1753.5-2349. The magenta line is the significance level averaged over the 66 months of survey; the blue line is the best significance.

Follow-up observations. We analysed all the available soft X-ray archival observations whose field of view covers the position of the BAT source candidates, starting from the huge set of SwiftXRT observations, many of which were performed for this purpose. For the BAT positions not covered by XRT observations, we searched for pointed archival observations with other X-ray instruments (Beppo-SAX, ASCA, Newton-XMM, Chandra, ROSAT). In each soft X-ray image, we searched for sources above an appropriate rate threshold within the a 6 arcmin radius error circle (99.7\% confidence level for a source detection at 4.8 standard deviations, [7]). The rate thresholds were chosen for each satellite in order to allow us to associate either faint or very absorbed sources with the BAT detection. With this method we expect a negligible number of spurious association, as evaluated performing a search for sources above the rate threshold in a large sample of GRB followup fields. Finally, the identification of the soft X-ray counterpart was performed by searching in the $\operatorname{SIMBAD}^{1}$ and $\mathrm{NED}^{2}$ databases within the soft X-ray error box.

Catalogue match. To find an association for the BAT excesses not associated with the follow-up method, we adopted the following strategy. We compiled a list of possible counterparts merging the following catalogues:

- high and low mass X-ray binaries, cataclysmic variables, supernova remnants and pulsars, Seyfert galaxies, unclassified AGNs, cluster of galaxies, interacting galaxies, LINERs, and $\boldsymbol{\gamma}$-ray sources, whose lists were extracted from the SIMBAD database on January 2010;

- the Roma-BZCAT [10];

- the ROSAT All Sky Survey (RASS) Bright source catalogue [11].

Then we performed a cross correlation of the BAT position with the positions of the sources in this list. With an association radius of 6.8 arcmin, we expect 2.5 spurious associations.

The final catalogue contains 1350 sources with an associated counterpart (1100 with the follow-up method and 250 with the catalogue match method). Figure 4 shows a comparison of

\footnotetext{
${ }^{1}$ http://simbad.u-strasbg.fr/simbad/

${ }^{2}$ http://nedwww.ipac.caltech.edu/
} 


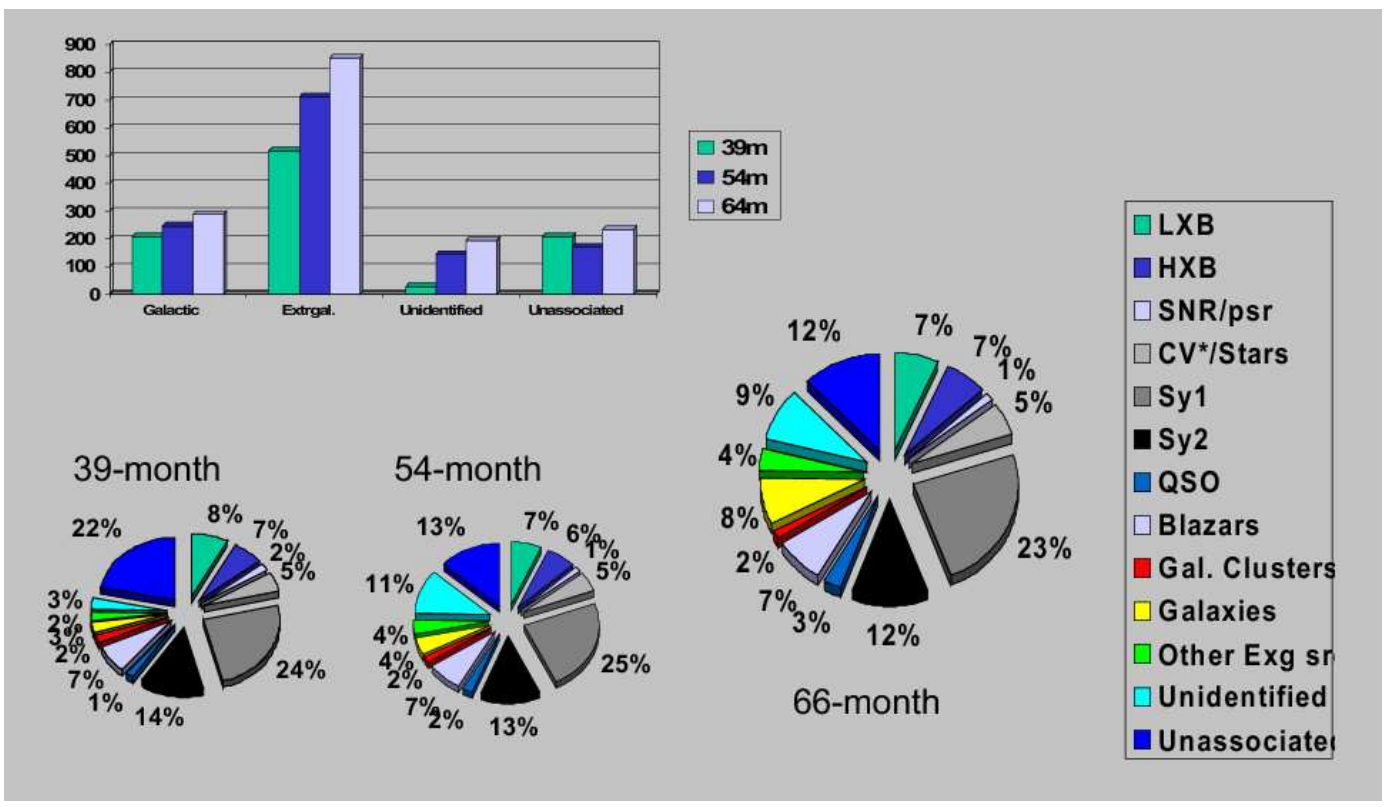

Figure 4: 66-month catalogue source class distribution compared to previous PBC catalogues

the 66-month PBC catalogue with the previous PBC catalogues. The histogram shows how the number of sources has increased with time (unidentified sources are sources associated to a softer counterpart whose nature has not yet been determined; unassociated sources are BAT sources with no observed X-ray counterpart). The three pie distributions show that the brakdown of the PBC sources into different source classes for the three PBC catalogues is mostly unchanged.

\section{BAT and ISGRI}

Figure 5 shows a comparison of the 66-month PBC catalogue with the list of ISGRI sources reported in the General Reference Catalog ${ }^{3}$ V.31. The INTEGRAL observing strategy has favoured for the first years of mission the observartion of the Galactic plane. On the contrary, the BAT observing plan follows the occurrence of GRBs and thus covers the sky almost uniformly. Together with the larger filed of view, this reflects in BAT being much more efficient than ISGRI in detecting extragalactic sources out of the Galactic plane. On the other hand, ISGRI is still more efficient in detecting Galactic sources, favoured both by the deeper exposure and by the smaller PSF, that allows it to resolve more sources in crowded fields. We have verified that the few X-ray binaries detected with BAT and not with ISGRI are either in regions with low ISGRI exposure or they are transient sources.

\section{BAT and Fermi}

We have searched for associations between the 66-month catalogue and the Fermi-LAT cata-

\footnotetext{
${ }^{3}$ http://www.isdc.unige.ch/integral/data/catalog
} 


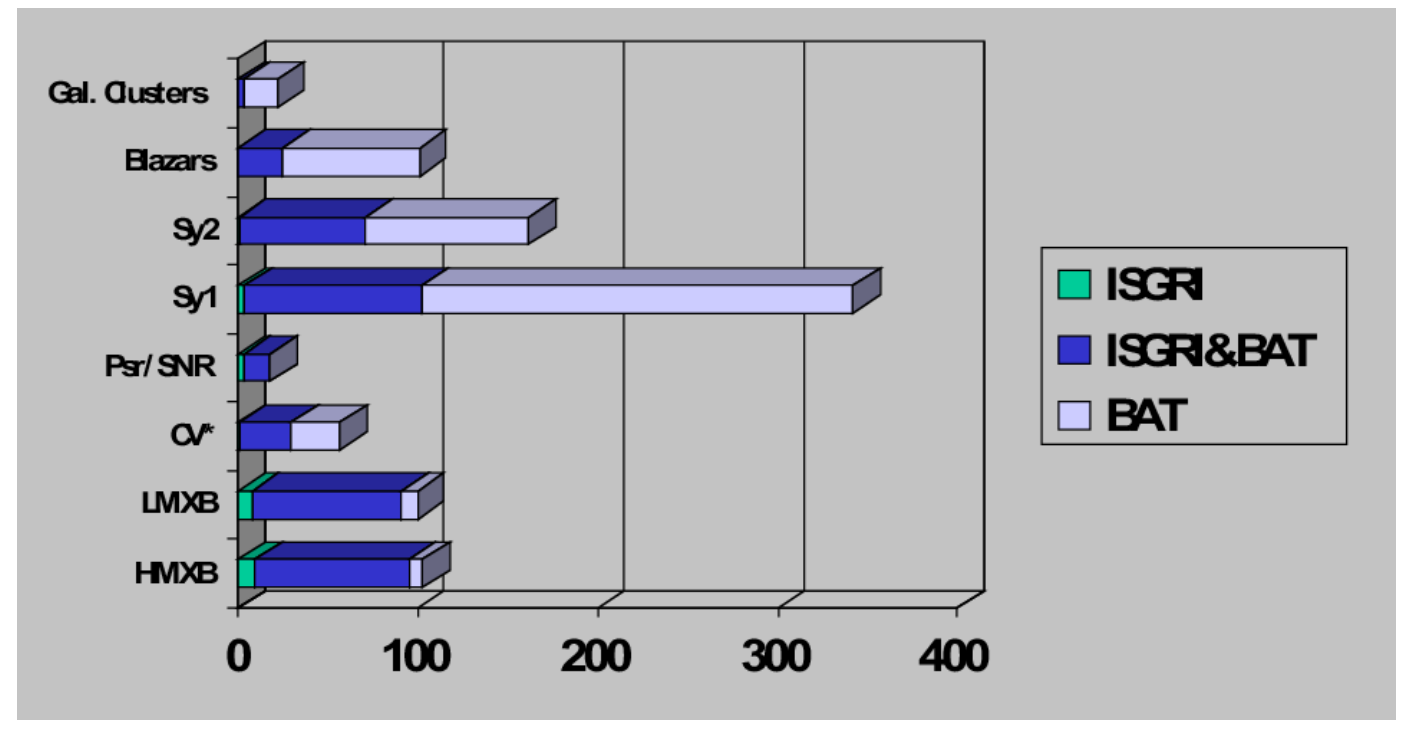

Figure 5: 66-month catalogue source class distribution compared to previous PBC catalogues.

logue [12], by cross-correlating the position of the sources seen by the two telescopes within their 95\% error circles. We find 67 positional coincidences (44 blazars, 4 Seyfert galaxies, 1 interacting galaxy, three high mass X-ray binaries and 4 pulsars/supernova remenants). Moreover, we also find 14 BAT sources whose counterpart lies within the error box of Fermi sources with different or no association.

\section{References}

[1] S. D. Barthelmy, L. M. Barbier, J. R. Cummings, et al., Space Science Reviews 1204, 143 (2005).

[2] N. Gehrels, G. Chincarini, P. Giommi, et al. ApJ 611, 1005 (2004)

[3] C. B. Markwardt, J. Tueller, G. K. Skinner, N. Gehrels, S. D. Barthelmy, \& R. F. Mushotzky, ApJ 633, L77 , (2005)

[4] M. Ajello, J. Greiner, G. Kanbach, A. Rau, A.W. Strong, J.A. Kennea, AJ 678, 102 (2008)

[5] J. Tueller, R.F. Mushotzky, S. Barthelmy, J.K. Cannizzo, N. Gehrels, C.B. Markwardt, G.K. Skinner, and L.M. Winter,ApJ 681, 113 (2008)

[6] J. Tueller, W.H. Baumgartner, C.B. Markwardt, et al, ApJS 186, 378 (2010)

[7] A. Segreto, G. Cusumano, C. Ferrigno, et al., $A \& A$ 510, 47 (2010)

[8] G. Cusumano, V. La Parola, A. Segreto, et al., A\&A 510, A48 (2010)

[9] G. Cusumano, V. La Parola, A. Segreto, et al., A\&A 524, A64 (2010)

[10] E. Massaro, P. Giommi, C. Leto, P. Marchegiani, A. Maselli, M. Perri, S. Piranomonte, S. Sclavi A\&A 495, 691 (2009)

[11] W. Voges, B. Aschenbach, T. Boller, et al., A\&A 349, 389 (1999)

[12] A.A. Abdo, M. Ackermann, M. Ajello et al. ApJS 187, 460 (2010) 